\title{
Brain biopsy in the management of focal encephalitis
}

\author{
N E Anderson, E W Willoughby, B J L Synek, M C Croxson, G L Glasgow
}

Auckland Hospital, of Neurology

NE Anderson

E W Willoughby

G L Glasgow

Department of

Pathology

B J L Synek

Department of

Virology

M C Croxson

School of Medicine,

University of

Auckland, Auckland,

New Zealand

Department of

Medicine

EW Willoughby

Department of

B J L Synek

Dr Anderson, Department of

Hospital, Park Road,

Auckland 1, New Zealand.

Received 30 July 1991

and in revised form

18 February 1991.
Auckland Department

Pathology

Correspondence to:

Neurology, Auckland

Accepted 28 February 1991

\begin{abstract}
The value of a brain biopsy in diagnosis and management of suspected herpes simplex encephalitis was studied in 29 patients (16 prospectively and 13 retrospectively). The biopsy showed herpes simplex encephalitis in eight, culture-negative encephalitis in 14, and was normal in three patients. It provided an alternative diagnosis in four patients, for two of whom curative treatment was available. The biopsy was complicated by a fatal intracranial haemorrhage in one patient. The low yield of alternative diagnoses suggests that a brain biopsy is not justified in the routine investigation of focal encephalitis.
\end{abstract}

The hallmarks of herpes simplex encephalitis (HSE) are its severity and signs of focal brain involvement, ${ }^{1}$ but the clinical features do not reliably distinguish HSE from other diseases. ${ }^{12}$ Detection of herpes simplex virus (HSV) in brain tissue is the most specific method of establishing the diagnosis ${ }^{3}$ and many authorities advocate brain biopsy in patients with a severe focal encephalitis. ${ }^{245}$ Others believe that a biopsy is not justified in every patient. ${ }^{67}$

Although antiviral therapy is often started before the diagnosis is established, confirmation of HSE by biopsy has two potential advantages. First, it may disclose a treatable disease other than HSE. ${ }^{2}$ Secondly, a full course of antiviral treatment may be avoided if it is negative. This was an important argument for biopsy when the only available antiviral

Table 1 Clinical features

\begin{tabular}{lcc}
\hline & Prospective & Retrospective \\
\hline Number & 16 & 13 \\
Male:Female & $8: 8$ & $4: 9$ \\
Age (years) Range & $0 \cdot 6-74$ & $3-66$ \\
$\quad$ Median & 25 & 25 \\
Duration symptoms before biopsy (days) & & \\
$\quad$ Range & $1-28$ & $0 \cdot 5-36$ \\
$\quad$ Median & 4 & 8 \\
Clinical features & & 13 \\
Altered mental state & 15 & 11 \\
Fever & 15 & 8 \\
Headache & 9 & 8 \\
Meningism & 6 & 5 \\
Seizures & 9 & 12 \\
Focal hemisphere symptoms, signs & 11 & $0-850$ \\
CSF Leukocytes ( $10^{6} /$ l) & & 22 \\
Range & $0-217$ & \\
Median & 22 & $0 \cdot 13-0 \cdot 99$ \\
CSF Protein (g/l) & & $0 \cdot 50$ \\
Range & $0 \cdot 20-1 \cdot 50$ & 10 \\
Median & $0 \cdot 46$ & 5 \\
Focal EEG abnormality & 11 & \\
Focal CT scan abnormality & 4 & \\
\hline
\end{tabular}

drugs had significant side effects. ${ }^{8}$ Acyclovir is not only a more effective treatment of HSE but is also relatively free of toxicity. ${ }^{9}$ Hence, apart from the cost of therapy the duration of treatment is no longer an important issue in determining if a biopsy helps in the management of HSE.

The aim of this study was to obtain more information about the place of biopsy in the diagnosis of acute focal encephalitis and to determine how the biopsy influenced management. Results of a prospective investigation of a more consistent approach to biopsy were compared with results for patients who had presented with focal encephalitis during the preceding four years.

\section{Patients and methods}

A prospective study of brain biopsy in patients with suspected HSE was started in October 1982 and completed in June 1985. Physicians at Auckland Hospital were encouraged to refer all patients with suspected HSE to the Neurology Department which is the only specialist neurological unit for a population of 1000000 . The decision to obtain a biopsy in an individual patient was made by the patient's neurologist with the informed consent of the patient, or the next of kin if the patient was unable to provide consent. Decisions about treatment were made by the attending neurologist so that the biopsy did not delay the institution of what was considered to be appropriate therapy.

Patients had combinations of fever, headache, altered mental state, seizures and meningism, and either focal signs or a focal abnormality on the EEG or CT brain scan (table 1). Patients were not considered for biopsy if 1) there was an obvious cause for the encephalitis (for example, mumps, infectious mononucleosis); 2) the clinical features were those of brain stem encephalitis; 3) they were aged less than six months; or 4) the CT scan suggested a subdural empyema, abscess, tumour, haemorrhage or infarct. All patients had an EEG. All but two patients had a CT scan before the biopsy; both of those had definite HSE on biopsy.

The biopsy was obtained by burr hole or craniectomy at a site based on the location of the focal abnormality on examination, CT scan, or EEG. The surgeon was asked to include leptomeninges, cerebral cortex and white matter in the specimen. A portion was homogenised and inoculated into human diploid fibroblast- cell cultures which were observed for four weeks before being discarded as negative. Only patients in whom HSV was 
Table 2 Total number of patients with suspected focal encephalitis, number referred to Neurology Department and number biopsied

\begin{tabular}{lllll}
\hline & $\begin{array}{l}\text { Duration } \\
\text { (months) }\end{array}$ & $\begin{array}{l}\text { Total presenting to } \\
\text { Auckland Hospital }\end{array}$ & $\begin{array}{l}\text { Referred to } \\
\text { Neurology }\end{array}$ & Biopsy \\
\hline & & & $(\%)$ & $(\%)$ \\
Prospective & 33 & 27 & $20(74)$ & $16(59)$ \\
Retrospective & 48 & 26 & $20(77)$ & $13(50)$ \\
\hline
\end{tabular}

cultured from the brain biopsy were regarded as having HSE. Other portions were fixed for histopathology and, in some cases, for electron microscopy and culture for bacteria and fungi. Follow up information was obtained from the hospital notes and by contacting the patient's general practitioner.

Thirteen patients who had a brain biopsy during investigation of focal encephalitis from November 1978 to October 1982 were studied retrospectively (table 1 ). These patients were traced from the operating theatre records. Patients admitted with focal encephalitis during the retrospective and prospective studies but not referred to the Neurology Department were traced from hospital records.

\section{Results}

A higher number of patients with focal encephalitis presented each year during the prospective study than in the retrospective study (table 2). This was presumably due to a fluctuation in incidence, as the proportion of patients referred to the Neurology Department did not change. There was little difference in the proportion of patients who had a brain biopsy in the prospective and retrospective groups. The most common reasons for not obtaining a biopsy were improvement in the first 24 hours and the belief that the results of the biopsy would not alter management.

Culture of HSV from the biopsy confirmed a diagnosis of HSE in only eight of the 29 patients $(28 \%$ ) (table 3 ). All eight positive biopsies showed acute encephalitis, four with intranuclear inclusion bodies. In another 14 patients the biopsy showed acute encephalitis (two with inclusion bodies), but HSV was not cultured. There was no significant difference between the prospective and retrospective groups.

In four patients the biopsy provided an alternative diagnosis: subdural empyema, astrocytoma, acute cerebral ischaemia and a non-granulomatous lymphocytic meningitis which improved with anti-tuberculous therapy. Features that are unusual for HSE were present in three of these patients. Two patients (meningitis, subdural empyema) had symptoms for four to five weeks. The CT scan in the patient with a subdural empyema was done in 1981 and showed an area of low density in one temporal lobe but the subdural empyema was not visible. A modern CT scan- ner probably would have detected this disorder. The patient with an astrocytoma was afebrile and the CSF was normal. A nonenhancing area of low density in the left basifrontal lobe was seen on the CT scan. HSE was thought to be the most likely diagnosis because of a short history (48 hours) and rapidly progressive symptoms.

The biopsy was normal in three patients. In one of them a further history, as the patient's level of consciousness improved, suggested subarachnoid haemorrhage and angiography showed a basilar artery aneurysm. A three week delay between the onset of symptoms and referral from another hospital accounted for the absence of subarachnoid blood on this patient's CT scan. Another patient had coma, fever, left hemiplegia, seizures and white matter hypodensity on CT scan, but the biopsy did not include white matter. This patient died but a necropsy was not obtained. In the third patient with a normal biopsy, the specimen included meninges, cerebral cortex and subcortical white matter. Necropsy showed acute disseminated encephalomyelitis, but the subcortical white matter was relatively spared.

All but one of the patients with encephalitis confirmed on biopsy were treated with an antiviral drug. If the biopsy also showed inclusion bodies or HSV cultures were positive, treatment was continued for at least 10 days. When the biopsy showed encephalitis without inclusion bodies and viral cultures were negative, the management was more variable. Treatment was continued for 10 days or longer in four patients in the prospective study and for at least six days in the remainder. Five of the seven patients in whom the biopsy did not show encephalitis had been started on an antiviral drug before the results of the biopsy were known. The drug was stopped in two where another diagnosis was made but was continued in the other three.

Two serious complications were attributable to the biopsy. One patient developed an intracerebral and subdural haematoma at the site of the biopsy and died. Another patient developed a moderate-sized subdural haematoma at the biopsy site, but recovered. Small intracerebral and subdural haematomas at the site of the biopsy were seen on CT scans or at necropsy in three other patients but they probably did not contribute to the neurological deterioration. One patient had seizures with an epileptogenic EEG focus in the area of the biopsy one month later.

\section{Discussion}

Although the object of the prospective study was to adopt a more consistent approach to brain biopsy in patients with suspected HSE, the study had no obvious effect on the rates of

Table 3 Results of brain biopsy

\begin{tabular}{|c|c|c|c|}
\hline & $\begin{array}{l}\text { Prospective } \\
(n=16)\end{array}$ & $\begin{array}{l}\text { Retrospective } \\
(n=13)\end{array}$ & $\begin{array}{l}\text { Combined } \\
(n=29)(\%)\end{array}$ \\
\hline $\begin{array}{l}\text { Herpes simplex encephalitis (Abnormal histology }+ \text { positive HSV culture) } \\
\text { Encephalitis with inclusion bodies, negative viral cultures } \\
\text { Encephalitis, no inclusion bodies, negative viral cultures } \\
\text { Alternative diagnosis } \\
\text { Normal }\end{array}$ & $\begin{array}{l}4 \\
2 \\
6 \\
3 \\
1\end{array}$ & $\begin{array}{l}4 \\
0 \\
6 \\
1 \\
2\end{array}$ & $\begin{array}{l}8(28) \\
2(7) \\
12(41) \\
4(14) \\
3(10)\end{array}$ \\
\hline
\end{tabular}


referral to the Neurology Department or biopsy. A frequent explanation for failure to proceed to biopsy was the perceived safety of acyclovir and the belief that the biopsy would not alter management.

A diagnosis of HSE was confirmed by the biopsy in only $28 \%$ of the patients in this study. This compares with yields of $33-57 \%$ in previous reports. ${ }^{148910}$ Several factors may reduce the diagnostic yield of brain biopsy in HSE. HSV has a patchy distribution in the affected brain ${ }^{11}$ and may be missed even when EEG and CT are used as guides to an appropriate site for a biopsy. The processing of the tissue is another potential source of error. We relied on viral culture as the most sensitive method of demonstrating HSV. ${ }^{3}$ Other techniques such as immunofluorescent assay for HSV antigens in brain tissue have a lower yield of positive results ${ }^{7}$ but may be complementary. It is not possible to determine how many of the 14 patients with biopsy evidence of acute encephalitis actually had HSE with false negative cultures. That was probably the case for the two who had inclusion bodies and an apparent response to acyclovir suggests several others may have had HSE. The frequency of false negative biopsies in practice may be greater than previously suggested. ${ }^{4}$

Several disorders may present with a clinical picture similar to HSE. ${ }^{2}$ In this study, biopsy revealed a diagnosis other than encephalitis in $14 \%$ of patients. Alternative diagnoses were found in $22 \%$ of patients with suspected HSE in the four multicentre antiviral drug trials in the United States. In some of those patients the alternative diagnosis was provided by specimens other than the brain biopsy. ${ }^{2}$ Our experience suggests that modern imaging techniques should detect a number of the disorders that caused confusion in our study and in earlier studies (for example, subdural empyema, cerebral tumours). Although apparently not a problem in this study, a brain biopsy occasionally provides misleading information which results in inappropriate treatment. $^{12}$

Failure to confirm a diagnosis of HSE did not have a major effect on treatment. In the prospective study, acyclovir usually was continued for 10 days or more if the biopsy showed encephalitis whether cultures for HSV were positive or negative because it was recognised that false-negative brain cultures may occur ${ }^{14}$ and that the treatment was relatively free of side-effects. Antiviral treatment was continued in three of the seven patients in whom the biopsy did not show encephalitis because it was thought that encephalitis may have been missed by the biopsy. A curative alternative treatment was available for two of the four patients in whom the biopsy revealed an alternative diagnosis (lymphocytic meningitis and subdural empyema).

In most reports brain biopsy has had a morbidity of $2 \%$ or less and a negligible mortality. ${ }^{249}$ Other authors have reported a higher morbidity and mortality. ${ }^{13}$ Our experience confirms that brain biopsy in patients with focal encephalitis is not always a benign procedure. The risk of long-term complications after a biopsy is unknown, but may include epilepsy and cognitive abnormalities. ${ }^{14}$

In this study, we believe that brain biopsy was only marginally helpful in the management of patients with suspected HSE and it was associated with significant morbidity. Although the small number of patients means that our conclusions must be tentative, we believe that the yield of alternative diagnoses is insufficient to justify a brain biopsy as a routine investigation in focal encephalitis. If the clinical, CSF, EEG and CT findings suggest HSE, it is reasonable to treat the patient with acyclovir without confirmation of the diagnosis by biopsy. CSF examination and CT should detect most of the treatable disorders that have been confused with HSE. Magnetic resonance imaging shows changes that are characteristic but not pathognomonic of $\mathrm{HSE}^{15}$ and will also be helpful in excluding other lesions. Measurement of HSV antibody titres in serial CSF specimens can provide retrospective confirmation of the diagnosis of HSE in many patients, ${ }^{3}$ while detection of HSV antigens or DNA in CSF may be rapid, sensitive and specific methods of diagnosing HSE in the future. ${ }^{1617} \mathrm{~A}$ brain biopsy should be considered for patients in whom the presenting features are not characteristic of HSE or if progress during treatment with acyclovir is not satisfactory.

We are grateful for the support of the physicians and neurosurgeons of the Auckland Hospital Board who were involved in the management of the patients who have been reported.

1 Whitley RJ, Soong S-J, Linneman C, et al. Herpes simplex encephalitis. Clinical assessment. JAMA 1982;247: 317-20.

2 Whitley RJ, Cobbs CG, Alford CA, et al. Diseases that mimic herpes simplex encephalitis. Diagnosis, presentation, and outcome. $J A M A$ 1989;262:234-9.

3 Nahmias AJ, Whitley RJ, Visintine AN, Takei Y, Alford CA. Herpes simplex virus encephalitis: laboratory evaluations and their diagnostic significance $J$ Infect Dis ations and their

4 Whitley RJ, Soong S-J, Hirsch MS, et al. Herpes simplex encephalitis. Vidarabine therapy and diagnostic problems. $N$ Engl J Med 1981;304:313-8.

5 Hanley DF, Johnson RT, Whitley RJ. Yes, brain biopsy should be a prerequisite for herpes simplex encephalitis treatment. Arch Neurol 1987;44:1289-90.

6 Fishman RA. No, brain biopsy need not be done in every patient suspected of having herpes simplex encephalitis. Arch Neurol 1987;44:1291-2.

7 Kennedy PGE. A retrospective analysis of forty-six cases of herpes simplex encephalitis seen in Glasgow between 1962 and 1985. QJ Med 1988;68:533-40.

8 Whitley RJ, Soong S-J, Dolin R, et al. Adenine arabinoside therapy of biopsy-proved herpes simplex encephalitis National Institute of Allergy and Infectious Diseases Collaborative

9 Whitley RJ, Alford CA, Hirsch MS, et al. Vidarabine versus acyclovir therapy in herpes simplex encephalitis. $N$ Engl $J$ Med 1986;314:144-9.

10 Williams BB, Levine DP, Lerner AN. Course and sequelae in 51 patients undergoing brain biopsies for suspected herpes simplex virus, type I encephalitis (HSVE). Clin Res 1982;30:778A

11 Landry ML, Booss J, Hsiung GD. Duration of vidarabine therapy in biopsy-negative herpes simplex encephalitis. $J A M A$ 1982;247:332-4.

$12 \mathrm{Kohl}$ S, James AR. Herpes simplex virus encephalitis during childhood: importance of brain biopsy diagnosis. J Pediat 1985;107:212-5.

13 Kaufman $\mathrm{HH}$, Catalano LW. Diagnostic brain biopsy: a series of 50 cases and a review. Neurosurgery 1979;4: 129-36.

14 Caplan LR. Brain biopsy in herpes simplex encephalitis. $N$ Engl J Med 1980;303:700.

15 Schroth G, Gawehn J, Thron A, Vallbracht A, Voigt K. Early diagnosis of herpes simplex encephalitis by MRI.

16 Lakeman FD, Koga J, Whitley RJ. Detection of antigen to herpes simplex virus in cerebrospinal fluid from patients with herpes simplex encephalitis. I Infect Dis 1987; 155:1172-8.

17 Powell KF, Anderson NE, Frith RW, Croxson MC. Noninvasive diagnosis of herpes simplex encephalitis. Lancet 1990;i:357-8. 